\title{
X-Ray-Standing-Wave Atom Location in Heteropolar Crystals and the Problem of Extinction
}

\section{Citation}

Patel, J. R., and J. A. Golovchenko. 1983. X-Ray-Standing-Wave Atom Location in Heteropolar Crystals and the Problem of Extinction. Physical Review Letters 50, no. 23: 1858-1861. doi:10.1103/physrevlett.50.1858.

\section{Published Version}

doi:10.1103/PhysRevLett.50.1858

\section{Permanent link}

http://nrs.harvard.edu/urn-3:HUL.InstRepos:29407049

\section{Terms of Use}

This article was downloaded from Harvard University's DASH repository, and is made available under the terms and conditions applicable to Other Posted Material, as set forth at http:// nrs.harvard.edu/urn-3:HUL.InstRepos:dash.current.terms-of-use\#LAA

\section{Share Your Story}

The Harvard community has made this article openly available.

Please share how this access benefits you. Submit a story.

Accessibility 


\title{
X-Ray-Standing-Wave Atom Location in Heteropolar Crystals and the Problem of Extinction
}

\author{
J. R. Patel and J. A. Golovchenko \\ Bell Laboratories, Murray Hill, New Jersey 07974 \\ (Received 15 April 1983)
}

\begin{abstract}
By monitoring the characteristic fluorescent radiation excited by $\mathrm{x}$-ray standing waves we have been able to distinguish the two different kinds of lattice sites in a heteropolar crystal of GaAs. The strong extinction effects that mask the position-dependent fluorescent signal were minimized by adopting a suitable detector geometry.

PACS numbers: $61.60 .+\mathrm{m}, 61.10 . \mathrm{Fr}$
\end{abstract}

One of the more fascinating aspects of dynamical Bragg diffraction of $x$ rays is the excitation of standing-wave fields inside the crystal. Direct experimental manifestations of standing-wave effects were evident in the discovery of anomalous transmission in the case of the Laue geometry by Borrmann ${ }^{1}$ and in studies of fluorescent scattering in the Bragg geometry initiated by Batterman. ${ }^{2,3}$ Experiments in the Bragg geometry (i.e., the diffracted beam exits the crystal through the same face that the incident beam enters) had the peculiar advantage that only a single standingwave-type eigenfunction is excited in the crystal and this field could be manipulated experimentally to probe atom sites in the diffracting crystal. It was later pointed out by Golovchenko, Brown, and Batterman ${ }^{4}$ that studies directly within the Bragg band $g a p^{5}$ could lead to comprehensive atom-location information. Subsequent work has demonstrated that both surface and near-surface impurities can be localized to approximately one hundredth of a lattice constant. ${ }^{6}$

It is the purpose of the following to demonstrate how recent progress in this field may be extended to the case of heteropolar crystals. In particular we, shall show that it is possible to distinguish the two different kinds of lattice sites from within the Bragg band-gap region. Hence, in this preliminary study we concentrate on distinguishing the different signals coming from the two kinds of (111) planes in a gallium-arsenide crystal. Since these are bulk atoms and not isolated to near-surface positions as in all previous latticelocation work, we are forced to adopt new procedures which provide insight into the nature of the extinction problem.

The standing-wave method of locating atoms is based upon the fact that as one tunes the angle between an incident beam and a set of Miller planes in the vicinity of the Bragg condition a strong reflected beam is excited that interferes with the incident $\mathrm{x}$ rays. This results in a standing-wave pattern with the local periodicity of the diffract- ing planes. The phase of this standing wave, relative to the Fourier component of the electron charge density responsible for the reflection, is obtained from the dynamical theory of diffraction. The theory predicts an angular band gap where the phase of the standing wave shifts continuously from being in phase with the charge-density component to being completely out of phase. Since the reflected and incident beam intensities are nearly equal, this is also a region where the modulation of the standing-wave field is complete. Outside the band-gap region the standing-wave phase is no longer a function of incidence angle and the only effect of increasing the angular deviation from the Bragg condition is a loss of reflected beam intensity and hence standing-wave modulation. The fluorescent $\mathrm{x}$-ray emission of atoms in the standing-wave field is very nearly proportional to the $\mathrm{x}$-ray intensity on the nuclei of the atoms. Thus, by monitoring the fluorescent intensity as a function of $\mathrm{x}$-ray incidence angle a curve characteristic of the atoms' positions will be obtained.

Figure 1 illustrates the principles discussed above. Shown is an idealized theoretical plot of the expected reflectivity and fluorescent yield for a (111) reflection in a zinc-blende structure like GaAs. There are two inequivalent (111) planes $A$ and $B$ as shown in the inset to Fig. 1, which also shows the corresponding fluorescent-yield curves $A$ and $B$ expected from atoms in each of these planes, respectively. It is important to note that these are the results expected if only a shallow layer of atoms near the surface contributes to the yield. In general, this will not be the case and extinction effects must be considered as well. We shall be forced to deal with this question shortly. Figure 1 contains only effects associated with standing-wave motion and modulation. Note that fluorescence from the position in plane $B$ has a large maximum within the band gap as the antinode of the standing-wave field passes through. The other position $A$ has a correspond- 


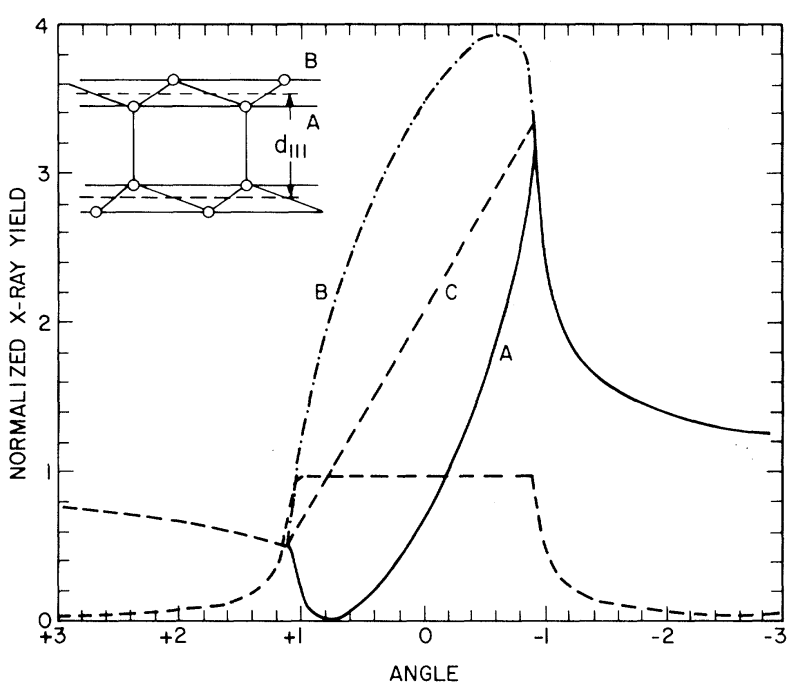

FIG. 1. Theoretical surface-fluorescence-yield curve for GaAs(111) with Mo $K \alpha$ x rays and no absorption. Curve $A$ is for atom positions on the plane $A$ and curve $B$ for the $B$-plane position shown in the inset, while curve $C$ is the average between the two. The Darwin reflection curve is shown dashed and the angular scale is normalized so that the band-gap region extends from 1 to -1 .

ing minimum at a symmetrically located angle due to the node passing through. It is interesting to see that outside of the band-gap region there is no difference expected between the fluorescent yields for these two particular sites. Finally, the curve labeled $C$ in Fig. 1 indicates the fluorescence expected from atoms equally distributed on both types of (111) planes. It should be clear that the essential detailed lattice-location information lies within the band-gap region.

The above discussion is essentially complete for the case where fluorescent signals emanate only from the immediate vicinity of the crystal surface. While this situation has been successfully achieved for impurity studies where atoms have been introduced by ion implantation, ${ }^{6,7}$ shallow diffusion, ${ }^{4}$ or wet chemistry, ${ }^{8,9}$ it is clearly not true for the study of bulk materials like GaAs. One must then cope with the effect of extinction mentioned earlier. Basically, the probing depth of the standing-wave- $\mathrm{x}$-ray field is a strong function of incidence angle and if signals can come from deep within the crystal then an angular scan of the fluorescent yield will be strongly modulated by this change in effective sample thickness. Unfortunately, the angular variation of this penetration is greatest within the band-gap region with the extinction length going through a mini-

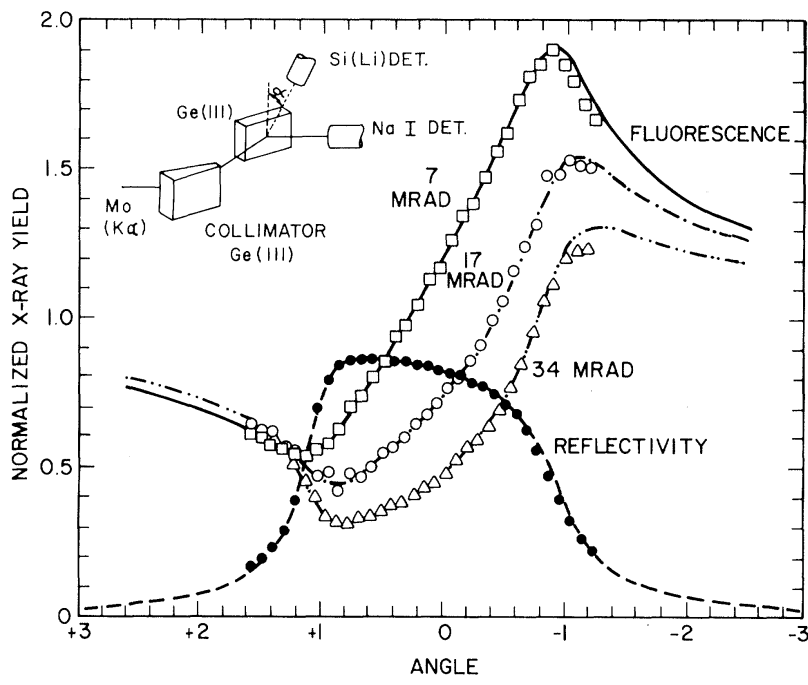

FIG. 2. Germanium $K \alpha$ fluorescence and reflectivity angular yield for (111) Bragg diffraction from germanium with Mo $K \alpha_{1}$ incident $\mathrm{x}$ rays. Fluorescence-yield curves were taken with the detector at the grazing angles to the surface indicated in milliradians.

mum directly in the middle of the gap. This is the reason why virtually all studies ${ }^{2,10}$ of bulk atom fluorescence reported in the literature until now show a strong dip directly at the center of the band gap which precludes the achievement of quantitative lattice-location information.

Stimulated both by Batterman's experiments ${ }^{2}$ and by the recent discovery of $\mathrm{x}$-ray evanescentwave emission ${ }^{11}$ we have attempted to limit the depth from which fluorescent signals may be detected by a careful choice of detector geometry. Because of technical details (e.g., problems with surface preparation) it has proved much simpler for us to demonstrate the nature of the reduction of extinction effects in the study of a germanium crystal. We therefore present results for germanium first and then discuss the closely related GaAs case.

A schematic of the experimental layout is contained in Fig. 2. The beam from a conventional $1-\mathrm{kW}$ fine-focus molybdenum $\mathrm{x}$-ray tube was collimated by an asymmetric (111) germanium crystal to an effective angular divergence of $\frac{1}{7}$ of the (111) natural reflection width. This beam was then intercepted by a (111) germanium crystal polished flat to $\frac{1}{4}$ wave over $4 \mathrm{~cm}^{2}$. Both the fluorescence and Bragg-reflected $x$-ray intensities were recorded as this second crystal was scanned in angle through the Bragg reflection region. The fluorescent $x$ rays were monitored with an ener- 
gy-dispersive $\mathrm{Si}(\mathrm{Li})$ detector mounted at a small grazing angle $\alpha$ to the surface in order to limit the depth from which signals were recorded. A similar detector-fluorescence geometry was employed by Batterman ${ }^{2}$ to enhance experimental detail outside the Bragg band-gap region.

Both the germanium $K \alpha$ fluorescent yield and the Bragg reflection curve are displayed in Fig. 2 for successively smaller grazing angles $\alpha$ to the surface. Clearly visible in the three fluorescent curves is the reduction in the effects of extinction as $\alpha$ is reduced from 34 to $7 \mathrm{mrad}$. At $7 \mathrm{mrad}$ the curve begins to resemble the ideal position signal, curve $C$ in Fig. 1. Using standard absorption coefficients we estimate that the depth from which the recorded fluorescence signal originates varies from $1.6 \mu \mathrm{m}$ at $\alpha=34 \mathrm{mrad}$ to $3290 \AA$ for the $7-\mathrm{mrad}$ detector setting. The latter nearly coincides with a calculated minimum extinction length at the center of the high-reflectivity region of $3030 \AA$. Further reduction in extinction can be expected as $\alpha$ is continually reduced. Virtually ideal position signals similar to those experimentally achieved for near-surface impurities can be expected for $\alpha \leqslant 0.3 \mathrm{mrad}$. The small detection solid angles as well as the requirements on surface flatness for such a geometry would certainly make such experiments impractical. However, Becker, Golovchenko, and Patel $^{11}$ have recently demonstrated that long before absorption at grazing angles reduces the escape depth sufficiently, a new phenomenon, $\mathrm{x}-$ ray evanescent wave emission, intervenes. This effect causes a drastic reduction in the depth from which fluorescence rays emerge for $\alpha \leqslant 4$ mrad, and future studies on a more intense $x$-ray source, such as a synchrotron, will surely be able to take advantage of it.

Before leaving the discussion of the germanium fluorescence we call attention to the drawn curves in Fig. 2 which represent the dynamical theory predictions that explicitly include not only the atomic plane positions but also extinction and absorption effects. Aside from use of the experimental data to determine the absolute orientation of the crystal surface, there are no adjustable parameters to the theory. Alternatively, one can obtain identical curves from more general energy-conservation arguments of the type used by Batterman; ${ }^{2}$ however, this approach has not been extended to distinguish the sites into which the absorptive energy is being dissipated. It is therefore inadequate for describing a case with inequivalent (111) planes such as the GaAs experi- ment which we consider next.

We have examined in some detail the $B$ surface of a dislocation-free GaAs crystal. In this orientation the arsenic atoms occupy the top (111) positions while the gallium atoms are below (see inset in Fig. 1). According to Fig. 1, we expect the angular fluorescent yield from arsenic to resemble curve $B$ and Ga to resemble curve $A$ in the absence of extinction effects. The experimental results with the $\mathrm{Si}(\mathrm{Li})$ detector at a $7-\mathrm{mrad}$ grazing angle are shown in Fig. 3. These results took $24 \mathrm{~h}$ to accumulate. The angular positions where the antinode of the standing-wave field passes through the As atoms is clearly distintinguished as the top of the large broad maximum in the As fluorescence. Similarly, the bottom of the dip in the $\mathrm{Ga}$ fluorescence corresponds to the node passing through the Ga-atom sites. The large difference between the $\mathrm{Ga}$ and $\mathrm{As}$ yields within the Bragg band-gap region is a clear demonstration of the high sensitivity to atomic positions inherent in these standing-wave measurements.

It is interesting to note that in terms of distortion due to extinction effects the As curves are already much superior to the 7-mrad germanium case. This is because the As $K \alpha$ fluorescent lines lie above the $\mathrm{Ga}$ absorption edge, which restricts the emergent signal to even shallower

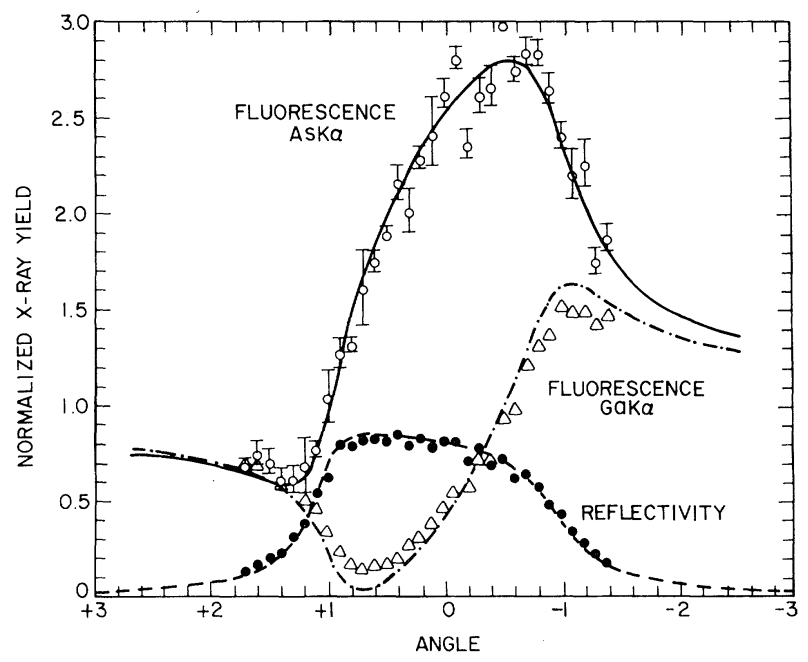

FIG. 3. Angular dependence of the fluorescence yield curves for $\mathrm{Ga}$ and $\mathrm{As} K \alpha$ and reflectivity for (111) Bragg diffraction from a GaAs crystal with incident Mo $K \alpha \times$ rays. The detector was located at a grazing angle of $7 \mathrm{mrad}$ with respect to the (111) crystal surface. The error bars for the Ga fluorescence are the width of the experimental points. 
depths than was the case for the previously described germanium experiment. Here the escape depth is calculated to be $1060 \AA$ which is only about $\frac{1}{3}$ of the minimum extinction length (3290 for GaAs). As a result the maximum As $K \alpha$ yield is just $17 \%$ below that expected from a situation where extinction is absent. The theoretical predictions including atom positions, extinction, and absorption are shown by the drawn curves in Fig. 3. Overall agreement is good, especially for the As atoms. The quantitative agreement in the case of $\mathrm{Ga}$ is less satisfactory. We suspect that this is partially due to some excess Ga fluorescence resulting from the reabsorption of As $K \alpha \mathrm{x}$ rays excited by the standing waves in the sample.

In conclusion, we have demonstrated that $\mathrm{x}$-ray standing waves can be used to probe inequivalent atomic sites in a heteropolar crystal. In order to isolate the position signal in this study of uniformly distributed atoms as a function of depth it was necessary to investigate and minimize the data distortion effects associated with extinction. Low counting rates made the present experiments somewhat difficult but this problem should be alleviated at synchrotron source facilities where hundredfold to thousandfold improvements may be anticipated. As a final comment, note that even for a homopolar crystal like germanium all atomic sites are not equivalent at the (111) crystal surface where it becomes meaningful to ask "in which (111) plane do the surface impurity atoms lie?" We trust that the results we have presented here will convince the reader that $\mathrm{x}$ ray-standing-wave studies of such surface impurities should be particularly illuminating.

We wish to thank R. N. Hall and W. Wagner for the dislocation-free germanium and galliumarsenide samples. Several discussions with B. W. Batterman on the extinction problem are gratefully acknowledged. We thank L. C. Kimerling for useful comments.

${ }^{1}$ G. Borrmann, Z. Phys. $\underline{42}, 157$ (1941), and $\underline{127}, 297$ (1950).

${ }^{2}$ B. W. Batterman, Phys. Rev. 133, A759 (1964).

${ }^{3}$ B. W. Batterman, Phys. Rev. Lett. 22, 703 (1969).

${ }^{4} \mathrm{~J}$. A. Golovchenko, W. L. Brown, and B. W. Batterman, Phys. Rev. B 10,4239 (1974).

${ }^{5}$ We refer to the angular region where no itinerant solutions to Maxwell's equations exist within the crystal as the Bragg band gap. This is the region normally associated with the high reflectivity characterized by the Darwin-Prins reflection curve. See R. W. James, Solid State Phys. 15, 55 (1963)。

${ }^{6}$ S. K. Andersen, J. A. Golovchenko, and G. Mair, Phys. Rev. Lett. 37, 1141 (1976).

${ }^{7}$ M. V. Kruglov, V. N. Shchemelev, and G. G. Kareva, Phys. Status Solidi (a) 46, 343 (1978).

${ }^{8}$ P. L. Cowan, J. A. Golovchenko, and M. F. Robbins, Phys. Rev. Lett. 44, 1680 (1980).

${ }^{9}$ J. A. Golovchenko, J. R. Patel, D. R. Kaplan, P. L. Cowan, and M. J. Bedzyk, Phys. Rev. Lett. $\underline{49}, 560$ (1982).

${ }^{10}$ P. Trucano, Phys. Rev. B 13 , 2524 (1976).

${ }^{11}$ R. S. Becker, J. A. Golovchenko, and J. R. Patel, Phys. Rev. Lett. 50, 153 (1983). 